\title{
Trasplante hepático durante la pandemia del SARS-CoV-2 (COVID-19). Revisión de la literatura
}

\author{
Hepatic transplant during SARS-CoV-2 (COVID-19) pandemic. A literature review
}

\begin{abstract}
Bernardo Pérez-González', Freyris C. Thomas-Lora1', Irma Hoyo², Nayelli C. Flores-García1, Erika F. Rodríguez-Aguilar ${ }^{3}$ Víctor M. Páez-Zayas ${ }^{4}$,Ernesto Márquez-Guillén ${ }^{1}$, Víctor Visag-Castillo4, Ignacio García-Juárez ${ }^{*}$ y Alan G. Contreras ${ }^{5}$

${ }^{1}$ Departamento de Gastroenterología, Instituto Nacional de Ciencias Médicas y Nutrición Salvador Zubirán, Ciudad de México, México; ${ }^{2}$ Servicio de Medicina Interna y Práctica Médica Grupal de Infectología, Centro Médico ABC, Ciudad de México, México; ${ }^{3}$ Unidad de Trasplante Hepático, Hospital Clínico, Universidad de Barcelona, Barcelona, España; ${ }^{4}$ Departamento de Trasplante de Órganos, Hospital General de México Dr. Eduardo Liceaga, Ciudad de México, México; ${ }^{5}$ Departamento de Trasplante de Órganos, Instituto Nacional de Ciencias Médicas y Nutrición Salvador Zubirán, Ciudad de México, México
\end{abstract}

\section{Resumen}

Antecedentes: En diciembre de 2019 se identificó en la ciudad de Wuhan, China, un nuevo beta coronavirus, el SARS-CoV-2, como agente causal de neumonía grave, conocida como COVID-19, lo cual ha provocado medidas estrictas de aislamiento, cierre de programas de trasplante hepático y la necesidad de modificar los protocolos de tratamiento. Objetivo: Documentar la información publicada sobre el impacto de la COVID-19 en la población con antecedente de trasplante hepático y establecer un protocolo de tratamiento. Método: Se buscaron en PubMed los términos MeSH "SARS-CoV-2", "COVID-19", "trasplante hepático" y "tratamiento". Resultados: Hasta el momento se ha demostrado en la población con trasplante hepático una mayor facilidad para adquirir el virus, sin una diferencia en la mortalidad al compararla con la población general. La inmunosupresión debe continuar, sin suspender los inhibidores de la calcineurina. Del tratamiento específico, los esteroides son los que han demostrado el mayor beneficio clínico y una disminución de la mortalidad. Conclusión: El trasplante hepático no se asocia de manera independiente a una mayor mortalidad. Otros factores, además del trasplante, deben tomarse en cuenta al momento de establecer la gravedad.

Palabras clave: COVID-19. Trasplante hepático. Tratamiento.

\begin{abstract}
Background: In December 2019, a new beta coronavirus, SARS-CoV-2, was identified in the city of Wuhan, China, as a causative agent of severe pneumonia, known as COVID-19, which has led to strict isolation measures, closure of liver transplantation programs and the need to modify treatment protocols. Objective: Document the information published so far on the impact of COVID-19 in the population with a history of liver transplantation and establish a treatment protocol. Method: MeSH terms were searched for "SARS-COV-2", "COVID-19", "liver transplantation" and "treatment". Results: Up to now, a greater ease in acquiring the virus has been shown in the liver transplant population, without a difference in mortality when compared to the general population. Immunosuppression should continue at the minimum tolerated levels, without suspending calcineu-
\end{abstract}

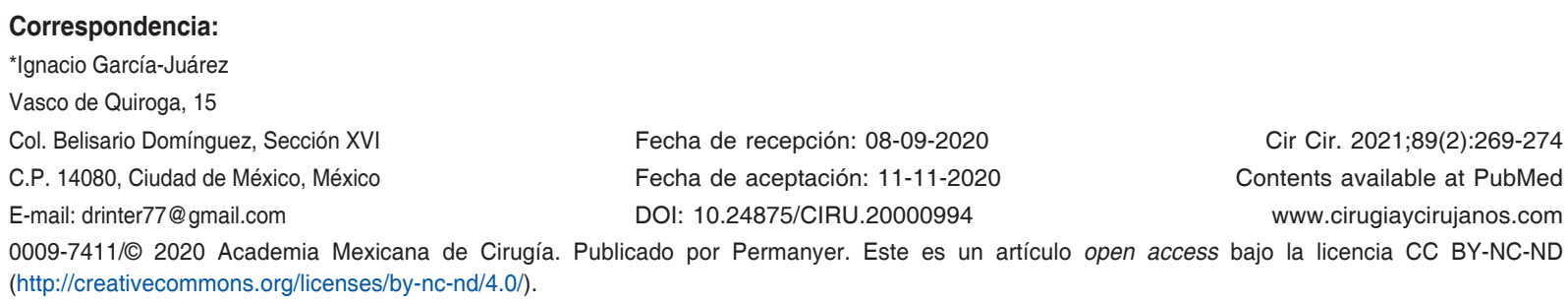

Cir Cir. 2021;89(2):269-274

Contents available at PubMed www.cirugiaycirujanos.com 0009-7411/C 2020 Academia Mexicana de Cirugía. Publicado por Permanyer. Este es un artículo open access bajo la licencia CC BY-NC-ND (http://creativecommons.org/licenses/by-nc-nd/4.0/). 
rin inhibitors. Of the specific treatment, steroids are those that have shown the greatest clinical benefit and decreased mortality. Conclusion: Liver transplantation is not independently associated with higher mortality. Factors other than transplantation must be taken into account when considering the risk of severity.

Key words: COVID-19. Liver transplant. Treatment.

\section{Introducción}

El grupo de los coronavirus pertenece a la familia Coronaviridae, del orden de los Nidovirales, virus compuestos por una estructura de RNA. Existen dos subfamilias que infectan al ser humano: alfa y beta'. La subfamilia alfa infecta las vías aéreas superiores y provoca síntomas leves, mientras que los virus beta, como el SARS-Co-V y el MERS-Co-V, son altamente patógenos y capaces de provocar un síndrome respiratorio agudo grave (SARS, por sus siglas en inglés)². En diciembre de 2019, en Wuhan, Hubei, China, se identificó un nuevo beta coronavirus, el SARS-CoV-2, asociado a un brote de neumonía grave que posteriormente la Organización Mundial de Salud (OMS) denominó COVID-19 (Coronavirus Disease 2019)³.

El espectro clínico de la COVID-19 puede ir desde leve, definido por la OMS por la ausencia de neumonía o hipoxia, hasta grave, cuando se presenta como neumonía con hipoxemia, la cual en ocasiones puede progresar a síndrome de insuficiencia respiratoria aguda $^{4-6}$.

Desde el comienzo de la pandemia, un tema de cuestionamiento frecuente ha sido el papel de la inmunosupresión en los pacientes receptores de trasplantes en cuanto a la influencia sobre la gravedad o el desenlace de la COVID-197,8. Un estudio demostró que los pacientes con inmunosupresión crónica por el trasplante tienen una mayor probabilidad de adquirir el virus, pero una menor probabilidad de desarrollar enfermedad grave ${ }^{9}$. El objetivo de este trabajo es realizar una revisión de la evidencia existente hasta el momento sobre el trasplante hepático durante la pandemia por COVID-19, tomando en cuenta el efecto de la inmunosupresión sobre el curso de la enfermedad, el ajuste de esta en caso de adquirir la infección, el protocolo a seguir con los pacientes en lista de espera y el futuro cercano de la reactivación del programa de trasplante.

\section{Método}

Se buscaron en PubMed los siguientes términos MeSH: "SARS-CoV-2", "COVID-19", "trasplante hepático" y "tratamiento", y posteriormente se identificaron y seleccionaron los artículos considerados más relevantes desde enero hasta agosto de 2020. No se realizó ningún tipo de análisis de datos.

\section{Manejo de la inmunosupresión en los pacientes receptores de trasplante hepático}

La población con trasplante hepático en general es considerada de alto riesgo para el desarrollo de enfermedades infecciosas graves, debido al uso de inmunosupresores de forma crónica, ya sea de anticuerpos monoclonales como el basiliximab, o de esteroides y antiproliferativos como el micofenolato de mofetilo y los inhibidores de la calcineurina; todo esto, dependiendo del tiempo transcurrido desde el trasplante y del grado de inmunosupresión necesario $0^{5,10}$. De inicio, en los pacientes asintomáticos no está recomendada la reducción de la inmunosupresión de manera profiláctica. Esto puede incrementar el riesgo de rechazo del injerto y no necesariamente traducirse en una menor gravedad si llegasen a contraer COVID-1911. En la actualidad, los datos sobre la COVID-19 en pacientes receptores de trasplantes es escasa, pero se sabe que la inmunosupresión prolongada incrementa la vulnerabilidad a adquirir la enfermedad y lleva a presentar una carga viral elevada en el momento del diagnóstico y una probabilidad mayor de desenlaces fatales ${ }^{12}$. Esto contrasta con la imposibilidad de suspender la inmunosupresión debido al riesgo de rechazo del injerto ${ }^{5,8,13}$. En un estudio de una cohorte multicéntrica de 151 pacientes receptores de trasplante hepático infectados por COVID-19, los cuales se compararon con 627 pacientes también infectados pero sin antecedente de trasplante, el desenlace principal evaluado, la mortalidad, no fue diferente entre ambos grupos, pero en el análisis de regresión lógistica multivariada se identificó un incremento de la mortalidad en el grupo de pacientes receptores de trasplante conforme se incrementaba la edad (odds ratio [OR]: 1.6 por cada año), y lo mismo con el incremento de la creatinina (OR: 1.57 por cada $1 \mathrm{mg} / \mathrm{dl}$ ) y la presencia de cáncer no hepático 
(OR: 18.3). Otros de los hallazgos en contra de los pacientes receptores de trasplante hepático fueron el mayor número de ingresos a las unidades de cuidados intensivos y la mayor necesidad de ventilación mecánica invasiva ${ }^{14}$. Tomando en cuenta los estudios publicados hasta el momento, los reportes de casos y la experiencia de cada centro, vemos que el comportamiento y el pronóstico de la COVID-19 en la población con trasplante hepático son similares a los de la población general15,16. En caso de adquirir la COVID-19 y tener antecedente de trasplante hepático, las recomendaciones dependerán de la gravedad del cuadro infeccioso. El primer cambio sugerido es reducir o suspender el micofenolato de mofetilo y mantener solo los inhibidores de la calcineurina en dosis tan bajas como sea posible. En los pacientes que reciben prednisona de manera crónica se deberá continuar con la dosis previa al ingreso, por vía oral o su equivalente por vía intravenosa, y al mismo tiempo evaluar el riesgo de insuficiencia suprarrenal secundaria y, en caso necesario, iniciar dosis de estrés con esteroides que tengan efecto mineralocorticoide ${ }^{11}$. Independientemente del manejo sobre la inmunosupresión, es importante saber que un $14-53 \%$ de los pacientes con COVID-19 pueden presentar alteraciones en la química hepática, especialmente con un patrón hepatocelular. La elevación suele ser transitoria, menor de cinco veces el valor superior normal y autolimitada. Esto no contraindica el inicio de tratamientos experimentales específicos para el virus ${ }^{17}$, pero sí obliga a una monitorización frecuente de la química hepática y a vigilar posibles interacciones farmacológicas, en particular con los inhibidores de la calcineurina y los inhibidores de m-TOR (mammalian target of rapamycin) ${ }^{11}$.

\section{Tratamiento de la COVID-19 en los pacientes receptores de trasplante hepático}

\section{Medidas generales}

La OMS recomienda que los pacientes con COVID-19 leve sean aislados y solo reciban tratamiento sintomático y de soporte, sin prescribir antibióticos profilácticos, y lo mismo para los pacientes con enfermedad moderada, pero en estos con vigilancia estrecha. Para los pacientes con enfermedad grave se recomienda hospitalizarlos, monitorizarlos continuamente, administrarles oxígeno suplementario y anticoagulación profiláctica, intermedia o terapéutica, según ameriten, e iniciar antibióticos profilácticos, desescalando o escalando diariamente de acuerdo con la evolución y el resultado de los cultivos ${ }^{4}$.

En cuanto al tratamiento específico, existen muchos medicamentos en investigación para el tratamiento de la COVID-19, todos con evidencia limitada y la gran mayoría aún considerados como experimentales.

\section{Corticosteroides}

Aunque en un principio fue controversial el uso de esteroides para los pacientes con COVID-19 grave, debido a un probable retraso en el aclaramiento del virus y una prolongación de la estancia hospitalaria ${ }^{18}$, actualmente se sabe que en los pacientes graves tienen un impacto en la disminución de la mortalidad a 28 días. El corticosteroide recomendado es la dexametasona a dosis de $6 \mathrm{mg} / \mathrm{dí}$ por 10 días $^{19}$, pero puede utilizarse su equivalente en metilprednisolona o hidrocortisona ${ }^{20}$.

\section{REMDESIVIR}

El remdesivir es un antiviral con acción directa, con efecto inhibitorio in vitro sobre la ARN polimerasa de Ios SARS-CoV 1 y 2, así como del MERS-CoV 21,22. Aunque aún no se encuentra aprobado por la Food and Drug Administration (FDA) de los Estados Unidos de América, existe permiso de emergencia para su uso en pacientes con COVID-19 grave $^{23,24}$. En los pacientes con enfermedad hepática 0 receptores de trasplante hepático se debe vigilar diariamente la química hepática durante la administración de remdesivir debido a que pueden elevar las transaminasas, y si estas se incrementa más de cinco veces sobre su valor normal se recomienda suspender el fármaco ${ }^{25}$.

\section{Plasma de pacientes convalecientes}

Este tipo de tratamiento no está recomendado por la OMS ${ }^{4}$. La Infectious Diseases Society of America solo lo recomienda para pacientes con COVID-19 grave y que se encuentren incluidos en un estudio clínico. Hasta el momento únicamente existe un ensayo clínico controlado que ha demostrado una probable mejoría clínica en pacientes graves ${ }^{26}$; las series de casos no han demostrado evidencia suficiente para evaluar un efecto en la mortalidad ${ }^{27}$. 


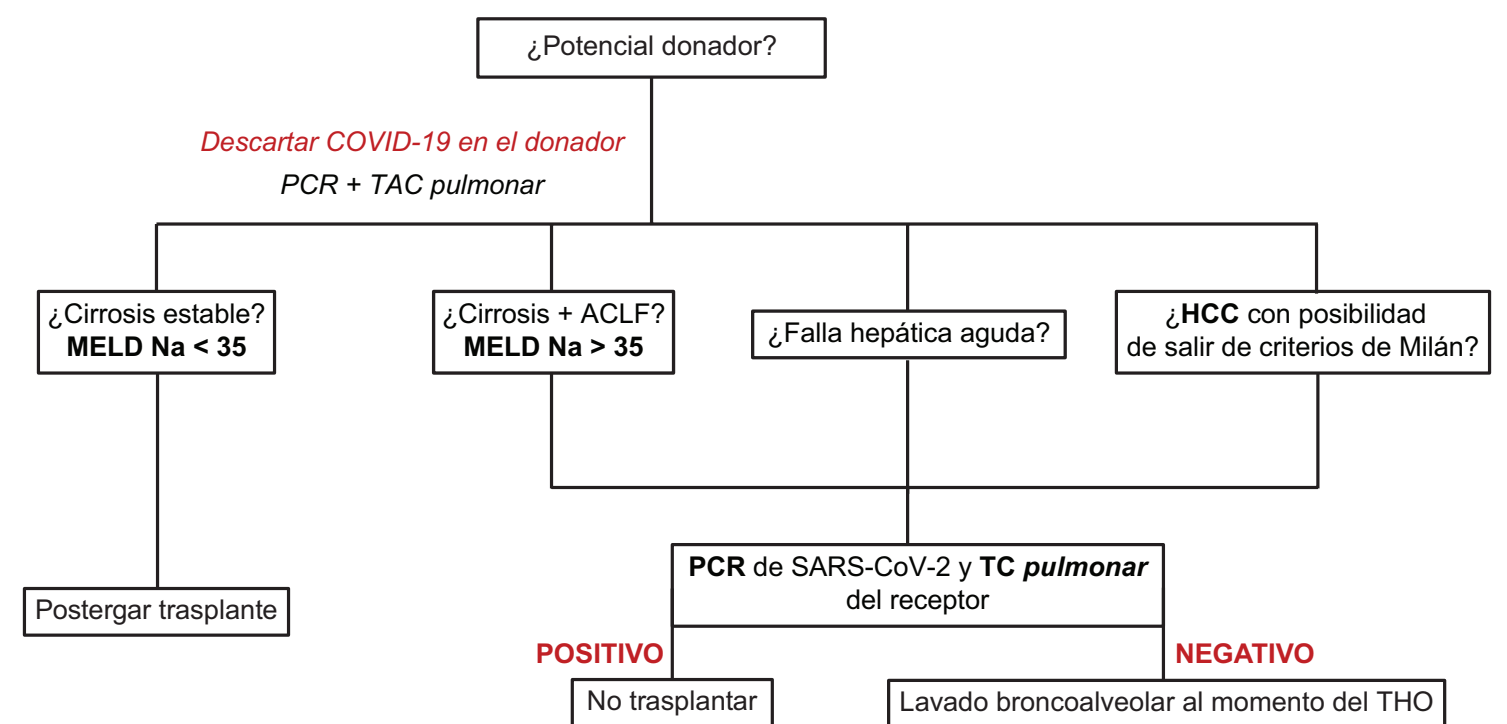

Apéndice: El donador no debió de haber tenido síntomas respiratorios al menos 14 días previos a la defunción, mismos criterios aplican para el receptor previo al THO

Figura 1. Algoritmo de trasplante hepático durante la pandemia de COVID-19. El donador no debió haber tenido síntomas respiratorios al menos en los 14 días previos a la defunción; mismos criterios aplican para el receptor. ACLF: Acute-on-Chronic Liver Failure; HCC: hepatocarcinoma; MELD Na: Model for End-Stage Liver Disease considerando la natremia; PCR: reacción en cadena de la polimerasa; TC: tomografía computada; THO: trasplante hepático ortotópico.

\section{HIDROXICLOROQUINA Y AZITROMICINA}

El uso de hidroxicloroquina o cloroquina no ha demostrado reducir el riesgo de intubación o de muerte en los pacientes hospitalizados ${ }^{28}$, y tampoco la tasa de negativización de la carga vira ${ }^{29}$. La asociación con azitromicina no ha demostrado disminuir la mortalidad, pero sí un incremento en el riesgo de paro cardiaco secundario a una prolongación del $\mathrm{QT}^{30}$, y la FDA ha retirado el permiso para su utilización ${ }^{31}$.

\section{TOCILIZUMAB}

El tocilizumab es un anticuerpo monoclonal que inhibe a la interleucina 6, la cual es uno de los principales mediadores de la inflamación provocada por la COVID-19. Al bloquear esta cascada de la inflamación en los pacientes con enfermedad grave y fiebre persistente, pudiera disminuir el daño orgánico secundario, pero hasta el momento la evidencia no es contundente y los estudios reportados son series de casos que, si bien en algunas de ellas se ha demostrado una mejoría en el patrón radiológico, disminución de la fiebre y de los marcadores inflamatorios, y de la probabilidad de requerir ventilación mecánica invasiva $^{32}$, en otras ocasiones no se ha observado ningún beneficio en la supervivencia a 14 días $^{33}$.

\section{Trasplante hepático durante la pandemia de COVID-19}

En este momento, la mayoría de los centros de trasplante en el mundo han cerrado sus programas debido a la pandemia de COVID-1934. Esto implica la muerte de muchos pacientes en lista de espera, la pérdida del seguimiento a los ya trasplantados y, en caso de reapertura del programa, el riesgo de contagio del personal de salud y su círculo cercano ${ }^{35}$. Podemos anticipar que los receptores de trasplante hepático se van a infectar, pero se sabe poco acerca de la transmisión del SARS-CoV-2 del donante al receptor y al personal médico ${ }^{36}$. Haga clic 0 pulse aquí para escribir texto. Las probabilidades de que el donante sea una potencial fuente de transmisión dependen no solo de los factores de exposición y del periodo de incubación (2-14 días), sino también de la viremia y de la viabilidad del virus en la sangre y en determinados órganos ${ }^{37,38}$. Durante la pandemia de SARS en 2002 se identificaron casos de infección en 
receptores de trasplante hepático y en los integrantes del equipo quirúrgico ${ }^{39}$.

\section{TRANSFUSIÓN DE HEMODERIVADOS DURANTE EL TRASPLANTE}

No se ha identificado que el coronavirus se transmita mediante la transfusión de hemoderivados, pero la FDA recomienda no recibir transfusiones de pacientes con síntomas respiratorios y el European Centre for Disease Prevention and Control recomienda esperar de 21 a 28 días para donar sangre tras haber tenido contacto con pacientes infectados o haber viajado a zonas de riesgo ${ }^{37}$.

\section{Pacientes en lista de espera}

Debido a que se desconoce la cinética del virus y existe la posibilidad de resultados falsos negativos del hisopado nasal40, se deben limitar los trasplantes hepáticos para los casos más urgentes (MELD [Model for End-stage Liver Disease] > 35, falla hepática aguda o carcinoma hepatocelular cercano a salir de los criterios de Milán) ${ }^{41}$. Todos los receptores deben ser tamizados para COVID-19, se les debe realizar una tomografía computada de pulmón y no se realizará el trasplante en los que estén infectados. En caso de que el donante haya estado en riesgo de exposición, pero sin presentar síntomas respiratorios ni fiebre previamente al deceso, pueden utilizarse sus órganos siempre y cuando no sean los pulmones o el intestino ${ }^{37}$ (Fig. 1).

\section{Agradecimientos}

Agradecemos a los diferentes profesionales y departamentos de gastroenterología que participaron en la búsqueda y la redacción de la información para el presente trabajo.

\section{Responsabilidades éticas}

Protección de personas y animales. Los autores declaran que para esta investigación no se han realizado experimentos en seres humanos ni en animales.

Confidencialidad de los datos. Los autores declaran que han seguido los protocolos de su centro de trabajo sobre la publicación de datos de pacientes.

Derecho a la privacidad y consentimiento informado. Los autores declaran que en este artículo no aparecen datos de pacientes.

\section{Conflicto de intereses}

No existe ningún conflicto de intereses por parte de los autores del presente artículo.

\section{Bibliografía}

1. Jin Y, Yang H, Ji W, Wu W, Chen S, Zhang W, et al. Virology, epidemiology, pathogenesis, and control of COVID-19. Viruses. 2020;12(4):372.

2. Wu A, Peng $Y$, Huang $B$, Ding $X$, Wang $X$, Niu $P$, et al. Genome composition and divergence of the novel coronavirus (2019-nCoV) originating in China. Cell Host Microbe. 2020;27(3):325-8.

3. World Health Organization Press Conference. The World Health Organization (WHO) has officially named the disease caused by the novel coronavirus as COVID-19. Disponible en: https://www.who.int/emergencies/ diseases/novel-coronavirus-2019.

4. World Health Organization clinical management of COVID-19, interim guidance. 27.05.2020. WHO/2019-nCoV/clinical/2020.5.

5. Guan W, Ni Z, Hu Y, Liang W, Ou C, He J, et al. Clinical characteristics of coronavirus disease 2019 in China. N Engl J Med. 2020:382:1708-20.

6. Chen N, Zhou M, Dong X, Qu J, Gong F, Han Y, et al. Epidemiological and clinical characteristics of 99 cases of 2019 novel coronavirus pneumonia in Wuhan, China: a descriptive study. Lancet. 2020;395:507-13.

7. Wang D, Hu B, Hu C, Zhu F, Liu X, Zhang J, et al. Clinical characteristics of 138 hospitalized patients with 2019 novel coronavirus-infected pneumonia in Wuhan, China. JAMA. 2020;323:1061-9.

8. Liu K, Fang Y-Y, Deng Y, Liu W, Wang M-F, Ma J-P, et al. Clinical characteristics of novel coronavirus cases in tertiary hospitals in Hubei Province. Chin Med J (Engl). 2020;133:1025-31.

9. Colmenero J, Rodríguez-Perálvarez M, Salcedo M, Arias-Milla A, Muñoz-Serrano A, Graus J, et al. Epidemiological pattern, incidence and outcomes of COVID-19 in liver transplant patients. J Hepatol. 2021;74:148-55.

10. Zhu N, Zhang D, Wang W, Li X, Yang B, Song J, et al. A novel coronavirus from patients with pneumonia in China, 2019. N Engl J Med. 2020;382:727-33.

11. COVID-19 (Coronavirus): FAQs for organ donation and transplantation. (Consultado el 28 de marzo de 2020.) Disponible en: https://wwwnc.cdc. gov/eid/article/27/2/20-4046_article.

12. American Society of Transplantation. Coronavirus Disease 2019 (COVID-19): frequently asked questions from transplant candidates and recipients. (Consultado el 28 de marzo de 2020.) Disponible en: https:// www.myast.org/coronavirus-disease-2019-covid-19-frequently-asked-questions-transplant-candidates-and-recipients.

13. Centers for Disease Control and Prevention (CDC). Implementation of mitigation strategies for communities with local COVID-19 transmission. (Consultado el 28 de marzo de 2020.) Disponible en: www.cdc.gov/COVID19.

14. Webb GJ, Marjot T, Cook JA, Aloman C, Armstrong MJ, Brenner EJ, et al. Outcomes following SARS-CoV-2 infection in liver transplant recipients: an international registry study. Lancet Gastroenterol Hepatol. 2020:5:1008-16.

15. Clinical Insights For Hepatology And Liver Transplant Providers During The Covid-19 Pandemic. American Association for the Study of Liver Diseases (AASLD). [citado 23 marzo 2020] Disponible en: www.aasld.org.

16. Michaels MG, La Hoz RM, Danziger Isakov L, Blumberg EA, Kumar D, Green M, et al. Coronavirus disease 2019: implications of emerging infections for transplantation. Am J Transplant. 2020;20:1768-72.

17. Pillai AA, Levitsky J. Overview of immunosuppression in liver transplantation. World J Gastroenterol. 2009;15:4225-33.

18. Li H, Chen C, Hu F, Wang J, Zhao Q, Gale RP, et al. Impact of corticosteroid therapy on outcomes of persons with SARS-CoV-2, SARSCoV, or MERS-CoV infection: a systematic review and meta-analysis. Leukemia. 2020;34:1503-11.

19. RECOVERY Collaborative Group, Horby P, Lim WS, Emberson JR, et al. Dexamethasone in hospitalized patients with COVID-19 - preliminary report. N Engl J Med. 2020 Jul 17:NEJMoa2021436. doi: 10.1056/NEJMoa2021436. Online ahead of print.

20. Bhimraj A, Morgan RL, Hirsch Shumaker A, Lavergne V, Baden L, ChiChung Cheng V. Infectious Diseases Society of America guidelines on the treatment and management of patients with COVID-19. Version 2.1.0. [citado 23 marzo 2020]. Disponible en: https://www.idsociety.org/practice-guideline/covid-19-guideline-treatment-and-management/.

21. Lo MK, Jordan R, Arvey A, Sudhamsu J, Shrivastava-Ranjan P, Hotard AL, et al. GS-5734 and its parent nucleoside analog inhibit Filo-, Pneumo-, and Paramyxoviruses. Sci Rep. 2017;7:43395.

22. Sheahan TP, Sims AC, Graham RL, Menachery VD, Gralinski LE, Case $\mathrm{JB}$, et al. Broad-spectrum antiviral GS-5734 inhibits both epidemic and zoonotic coronaviruses. Sci Transl Med. 2017;9:eaal3653.

23. Wang $Y$, Zhang D, Du G, Du R, Zhao J, Jin Y, et al. Remdesivir in adults with severe COVID-19: a randomised, double-blind, placebo-controlled, multicentre trial. Lancet. 2020;395:1569-78. [published correction appears in Lancet. 2020;395:1694]. 
24. Beigel JH, Tomashek KM, Dodd LE, Mehta AK, Zingman BS, Kalil AC, et al. Remdesivir for the treatment of COVID-19 - preliminary report. Reply. N Engl J Med. 2020;383:994.

25. U.S. Food and Drug Administration. Coronavirus (COVID-19) update: FDA warns of newly discovered potential drug interaction that may reduce effectiveness of a COVID-19 treatment authorized for emergency use. (Consultado el 5 de junio de 2020.) Disponible en: https:// www.fda.gov/news-events/press-announcements/coronavirus-covid-19-update-fda-warns-newly-discovered-potential-drug-interaction-may-reduce.

26. Li L, Zhang W, Hu Y, Tong X, Zheng S, Yang J, et al. Effect of convalescent plasma therapy on time to clinical improvement in patients with severe and life-threatening COVID-19: a randomized clinical trial. JAMA. 2020;324:460-70.

27. Duan K, Liu B, Li C, Zhang H, Yu T, Qu J, et al. Effectiveness of convalescent plasma therapy in severe COVID-19 patients. Proc Natl Acad Sci U S A. 2020;117:9490-6.

28. Geleris J, Sun Y, Platt J, Zucker J, Baldwin M, Hripcsak G, et al. Observational study of hydroxychloroquine in hospitalized patients with $\mathrm{CO}$ VID-19. N Engl J Med. 2020;382:2411-8.

29. Tang W, Cao Z, Han M, Wang Z, Chen J, Sun W, et al. Hydroxychloroquine in patients with mainly mild to moderate coronavirus disease 2019 open label, randomised controlled trial. BMJ. 2020;369:m1849.

30. Rosenberg ES, Dufort EM, Udo T, Wilberschied LA, Kumar J, Tesoriero $\mathrm{J}$, et al. Association of treatment with hydroxychloroquine or azithromycin with in-hospital mortality in patients with COVID-19 in New York State. JAMA. 2020;323:2493-502.

31. U.S. Food and Drug administration. Coronavirus (COVID-19) update: FDA revokes emergency use authorization for chloroquine and hydroxychloroquine. (Consultado el 15 de junio de 2020.) Disponible en: https:// www.fda.gov/news-events/press-announcements/coronavirus-covid-19-update-fda-revokes-emergency-use-authorization-chloroquine.
32. Alattar R, Ibrahim TBH, Shaar SH, Abdalla S, Shukri K, Daghfal JN, et al. Tocilizumab for the treatment of severe coronavirus disease 2019. J Med Virol. 2020;92:2042-49.

33. Price CC, Altice FL, Shyr Y, Koff A, Pischel L, Goshua G, et al. Tocilizumab treatment for cytokine release syndrome in hospitalized COVID-19 patients: survival and clinical outcomes. Chest. 2020;158:1397-408.

34. Guastalegname M, Vallone A. Could chloroquine/hydroxychloroquine be harmful in coronavirus disease 2019 (COVID-19) treatment? Clin Infect Dis. 2020;71:888-9.

35. Gautret P, Lagier J-C, Parola P, Hoang VT, Meddeb L, Mailhe M, et al. Hydroxychloroquine and azithromycin as a treatment of COVID-19: results of an open-label non-randomized clinical trial. Int $\mathrm{J}$ Antimicrob Agents. 2020;56:105949.

36. Cao B, Wang Y, Wen D, Liu W, Wang J, Fan G, et al. A trial of lopinavir-ritonavir in adults hospitalized with severe COVID-19. N Engl J Med. 2020;382:1787-99.

37. Holshue ML, DeBolt C, Lindquist S, Lofy KH, Wiesman J, Bruce H, et al. First case of 2019 novel coronavirus in the United States. N Engl J Med. 2020;382:929-36.

38. Bennardo F, Buffone C, Giudice A. New therapeutic opportunities for COVID-19 patients with tocilizumab: possible correlation of interleukin-6 receptor inhibitors with osteonecrosis of the jaws. Oral Oncol. 2020;106:104659.

39. Yao X, Ye F, Zhang M, Cui C, Huang B, Niu P, et al. In vitro antiviral activity and projection of optimized dosing design of hydroxychloroquine for the treatment of severe acute respiratory syndrome coronavirus 2 (SARS-CoV-2). Clin Infect Dis. 2020;71:732-9.

40. Harrison C. Coronavirus puts drug repurposing on the fast track. Nat Biotechnol. 2020;38:379-81.

41. Liverpool COVID-19 Interactions [Internet]. Liverpool COVID-19 Interactions. [Último acceso 29 ene 2021]. Disponible en: https://www.covid19-druginteractions.org 